Case Report

\title{
Chondrosarcoma of the anterior chest wall : surgical resection and reconstruction using a two layer polypropylene mesh and bone cement sandwich
}

\author{
Amol Amonkar', M undayat Gopalakrishnan ${ }^{2}$, AmithKiran Naik', \\ Vishwanath $\mathrm{S}^{4}$, Vimaladhithan ${ }^{5}$ \& Saquib Sultan ${ }^{6}$ \\ ${ }^{1,5,6}$ Post graduate, Department of Surgery, ${ }^{2} \mathrm{HOD}$ and Professor, ${ }^{3}$ Associate Professor, Department of Cardiothoracic and \\ vascular surgery, , ${ }^{4}$ Assistant Professor, Surgical Oncology, K. S. Hegde Medical Academy, Nitte University, Mangalore. \\ Correspondence \\ Amol Amonkar \\ Post graduate, Department of Surgery, K.S. Hegde M edical Academy, \\ Nitte University, Mangalore - 575 018, Karnataka, India. \\ M obile : +919886535829 E-mail : amonkaramol@gmail.com

\begin{abstract}
Primary malignant tumours of the chest wall are uncommon. Chondrosarcoma is the most common malignancy among them, the current therapy for chondrosarcoma requires adequate surgical excision. A 50 year old male presented with a swelling on the anterior chest wall, trucut biopsy of the swelling was reported as chondrosarcoma. Thorax computed tomography (CT) revealed a large mass lesion with the epicentre at the costal cartilage of the right $2^{\text {nd }}$ rib extending beyond the chest wall and musculature and protruding internally upto the upper lobe of the right lung, features likely of chondrosarcoma. Inorder to obtain disease free surgical margins, an enbloc resection of the tumour along with approximately $4 \mathrm{cms}$ of $1^{\text {st }}$ rib and $2^{\text {nd }}$ and $3^{\text {rd }}$ rib and reconstruction of the anterior chest wall was performed with a 2 layer polypropylene mesh and bone cement sandwich. The post-operative course was uneventful.The chest wall reconstruction with the two layer polypropylene mesh and bone cement provided the essential rigidity and stability to the chest wall.
\end{abstract}

Keywords: Chondrosarcoma, chestwall, reconstruction, two layer polypropylene mesh and bone cement sandwich.

\section{Introduction}

Primary malignant tumours of the chest wall are a heterogenous group of uncommon tumours developing from bone, cartilage or the soft tissues [1]. Chondrosarcoma is the most common primary malignancy of the anterior chest wall and the successful treatment of choice includes en-bloc removal of the tumour with surrounding tissue with a minimum clear margin of 2 to 6 $\mathrm{cm}$. Reconstruction of an oversized defect can be difficult and sometimes needs the use of a synthetic prosthesis. Hereby our clinical experience with a bone cement mesh reconstruction of a large Access this article online Quick Response Code

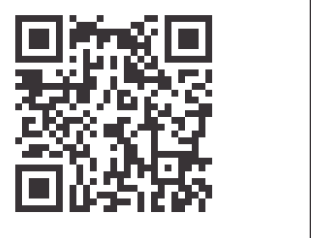
anterior chest wall defect is presented.

\section{Case Report}

A 50 year old male presented with a swelling on the right side of the anterior chest wall since 6 months which was insidious in onset and suddenly progressed in size since 1 month, with no other symptoms. On local examination of the chest wall a solitary swelling in the right side of the anterior chest wall measuring $18 \times 18$ $\mathrm{cm}$, which is hard in consistency and had well defined borders. Trucut biopsy was suggestive of chondrosarcoma .Thorax computed tomography revealed large mass lesion with the epicentre at the costal cartilage at the $2^{\text {nd }}$ rib with flocculant calcification centrally and soft tissue in the periphery extending beyond the chest wall and musculature and protruding upto the right upper lung features like that of chondrosarcoma. The patient was taken up for surgery under general anaesthesia with single lung ventilation and vascular access was obtained through the femoral vein. Intraoperatively it was found that the superior vena cava was compressed by the tumour, the other major vessels and the upper lobe of the lung were free of the tumour. The patient underwent an en-block resection of the tumour arising from $2^{\text {nd }}$ and $3^{\text {rd }}$ rib along 
with approximately $4 \mathrm{~cm}$ of $1^{\text {st }}$ rib.Chest wall defect was reconstructed with bone cement which was sandwiched between the polypropylene meshs. The edge of the mesh was anchored to the remaining ribs and sternum to stabilize the thoracic wall. It was then covered with a pectoralis major muscle,subcutaneous tissue and skin. The patient was extubated in the operation theatre and was maintaining well , the patient was shifted to the intensive care unit(ICU)for post-operative care. The post-operative period was uneventful.The histopathological report was suggestive of chondrosarcoma.

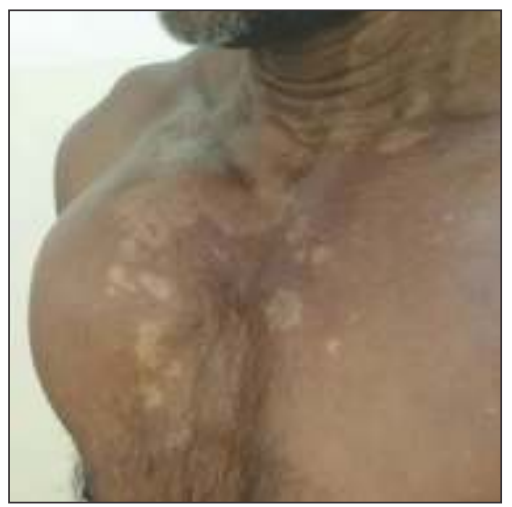

Figure 1 : a 50 year old male presented with a swelling measuring $18 \times 18 \mathrm{cms}$ with no other symptoms.

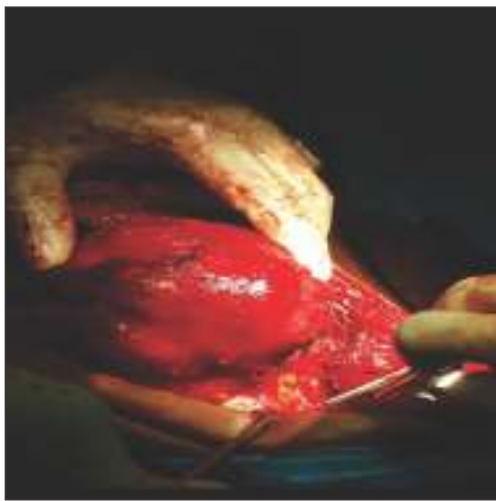

Figure 2 : Intra- operative findings showing a $14 \times 14 \mathrm{cms}$ tumour arising from the costal cartilage of the $2^{\text {nd }}$ rib extending upto the upper lobe of the right lung.

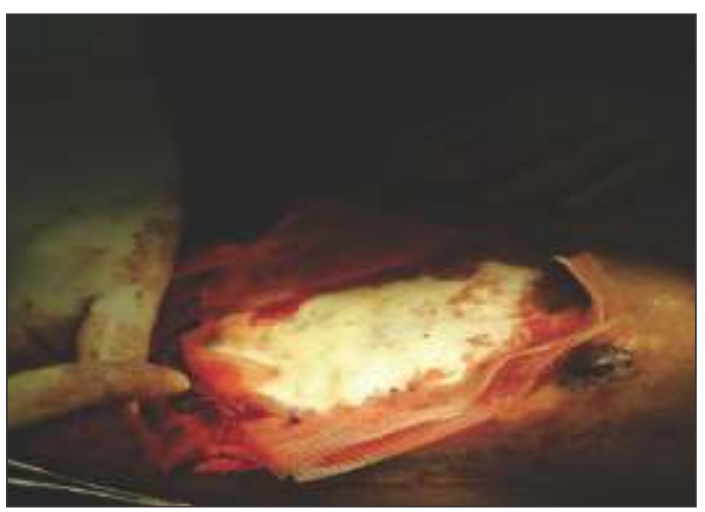

Figure 4 : A 2 layer polypropylene mesh with bone cement sandwich used for reconstruction of the chest wall.

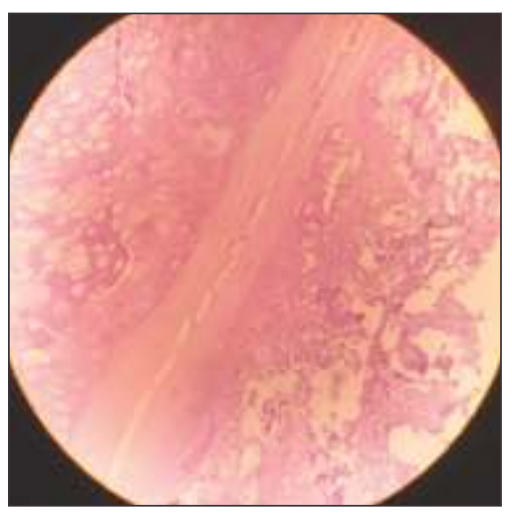

Figure 6 : Microscopy shows chondrocytes arranged in varying sized lobules separated by fibrous septa, tumour cells show m o d e r a t e I y hypercelluar and pleomorphism like irregular nuclei, atypical $\mathrm{m}$ i to s is. a n d binucleation.

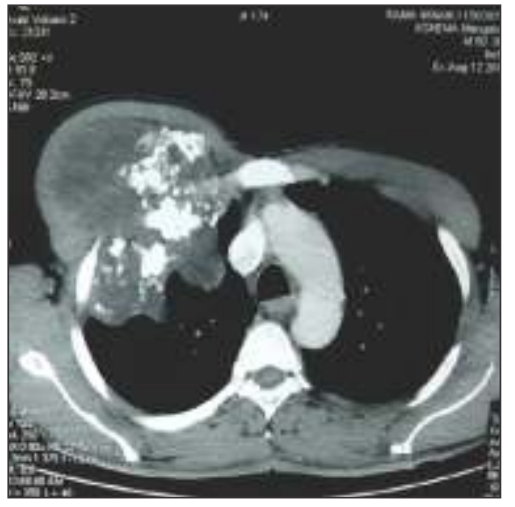

Figure 5 : Thorax CT showing a large mass lesion with epicentre at the costal cartilage at the $2^{\text {nd }}$ rib with flocculant calcification centrally and soft tissue in the periphery extending beyond the chest wall and musculature and protruding upto the right upper lung features like that of chondrosarcoma

\section{Discussion}

Chest wall tumours are rare and account for almost 0.5 $1 \%$ of the primary bone tumours, with chondrosarcoma being the most common subtype. It mostly occurs after the $6^{\text {th }}$ decade of life and has a slight male predominance[3,4]. It usually presents as a gradually growing,solid and fixed mass[3]. Occasionally with a concomitant chest pain, which is a sign of bad prognosis [3].Chest wall resection and reconstruction procedure requires adequate radical resection associated with maintenance of chest stability, adequate lung function and an acceptable cosmetic result. The purpose of adequate radical surgery is removal of the 
tumour with a wide disease free margin along with maintenance of chest wall stability[5].Inadequate tumour resection is associated with a high incidence of recurrence[5].The optimal incision should be 2 to $6 \mathrm{~cm}$ from the margin of the tumour in order to minimize the risk of local recurrence[6].Reconstruction is essential to maintain original respiratory function and protect other mediastinal organs, these reconstruction techniques include pedicled skin and muscle flaps, skin grafts and autologous bone transplants.The choice of reconstruction technique depends on the extent and localization of the defect. If the defect is less than $5 \mathrm{cms}$ then skeletal reconstruction is not necessary. M uscle flap reconstruction may be used in cases with sole upper half sternal resections. Resection of the lower half of sternum requires synthetic materials to stabilize rigid chest wall in order to protect the vital organs.[2-4].The prosthetic material used

\section{References}

1. Ersoz E et al.Chondrosarcoma of the anterior chest wall:surgical resection and reconstruction with titanium mesh. J thoracDis. 2014;6(10):230-233.

2. He $B$, Huang $Y, L i P, Y e X$, Lin $F$, Huang $L$ et al. A rare case of primary chondrosarcoma arising from the sternum: A case report. Oncology Letters. 2014.

3. 4. Locicero J S. general thoracic surgery. 5th ed. philadelphia: Lippincott Williamsand Wilkins; 2000.

4. Koto K, Sakabe T, Horie N, Ryu K, Murata H, Nakamura S et al. Chondrosarcoma from the sternum: Reconstruction with titanium mesh and a transverse rectus abdominis myocutaneous flap after subtotal sternal excision. M ed Sci M onit. 2012;18(10):CS77-CS81.

5. Incarbone, M.D. M, Pastorino, M.D. U. Surgical Treatment of Chest Wall Tumors. World J Surg. 2001;25(2):218-230.

6. Martini N, Huvos A, Burt M, Heelan R, Bains M, McCormack P et al. Predictors of survival in malignant tumors of the sternum. The Journal of Thoracic and Cardiovascular Surgery. 1996;111(1):96-106. during reconstruction should have sufficient rigidity to prevent paradoxical chest motion, must have adequate radiolucency to allow radiographic follow up and must be biocompatible structure which allows growth of fibrous tissue without causing any infection in it.[4].Hereby described a case of chondrosarcoma that needed an enbloc resection of the tumour with two layer polypropylene mesh and bone cement sandwich.

\section{Conclusion}

We conclude by stating that even large chest wall tumours can be successfully resected with a mulitidisciplinary team approach involving an oncosurgeon, cardiothoracic and vascular surgeon, plastic surgeon, intensivist, anaesthesiologist and good nursing care.Surgical resection is the mainstay of treatment of this tumour. Chemotherapy and radiotherapy have a limited role in the treatment of this tumour. 